\title{
On Finite Alphabets and Infinite Bases II: Completed and Ready Simulation ${ }^{\star}$
}

\author{
Taolue Chen ${ }^{1,2}$, Wan Fokkink ${ }^{1,3}$, and Sumit Nain ${ }^{4}$ \\ 1 CWI, Department of Software Engineering, PO Box 94079, \\ 1090 GB Amsterdam, The Netherlands \\ chen@cwi.nl \\ 2 Nanjing University, State Key Laboratory of Novel Software Technology, Nanjing, \\ Jiangsu, P.R. China, 210093 \\ 3 Vrije Universiteit Amsterdam, Department of Theoretical Computer Science, \\ De Boelelaan 1081a, 1081 HV Amsterdam, The Netherlands \\ wanf@cs. vu.nl \\ 4 Rice University, Department of Computer Science, 6100 S. Main Street, Houston, \\ TX 77005-1892, USA \\ sumitnain@yahoo.com
}

\begin{abstract}
We prove that the equational theory of the process algebra BCCSP modulo completed simulation equivalence does not have a finite basis. Furhermore, we prove that with a finite alphabet of actions, the equational theory of BCCSP modulo ready simulation equivalence does not have a finite basis. In contrast, with an infinite alphabet, the latter equational theory does have a finite basis.
\end{abstract}

\section{Introduction}

Labeled transition systems constitute a fundamental model of concurrent computation which is widely used in light of its flexibility and applicability. They model processes by explicitly describing their states and their transitions from state to state, together with the actions that produce them. Several notions of behavioral equivalence have been proposed, with the aim to identify those states of labeled transition systems that afford the same observations. The lack of consensus on what constitutes an appropriate notion of observable behavior for reactive systems has led to a large number of proposals for behavioral equivalences for concurrent processes.

Van Glabbeek [6] presented the linear time - branching time spectrum of behavioral preorders and equivalences for finitely branching, concrete, sequential processes. In this paper we focus on two semantics in this spectrum. A relation $R$ between processes is a simulation if $s_{0} R s_{1}$ and $s_{0} \stackrel{a}{\rightarrow} s_{0}^{\prime}$ implies $s_{1} \stackrel{a}{\rightarrow} s_{1}^{\prime}$ with $s_{0}^{\prime} R s_{1}^{\prime}$. Such a relation is a completed simulation if whenever $s_{0}$ cannot perform any transition, the same holds for $s_{1}$. It is a ready simulation if $s_{0}$ and $s_{1}$ can

\footnotetext{
* Partially supported by the Dutch Bsik project BRICKS (Basic Research in Informatics for Creating the Knowledge Society), 973 Program of China (No. 2002CB312002), and NNSFC (No. 60233010, No. 60273034, No. 60403014).
} 


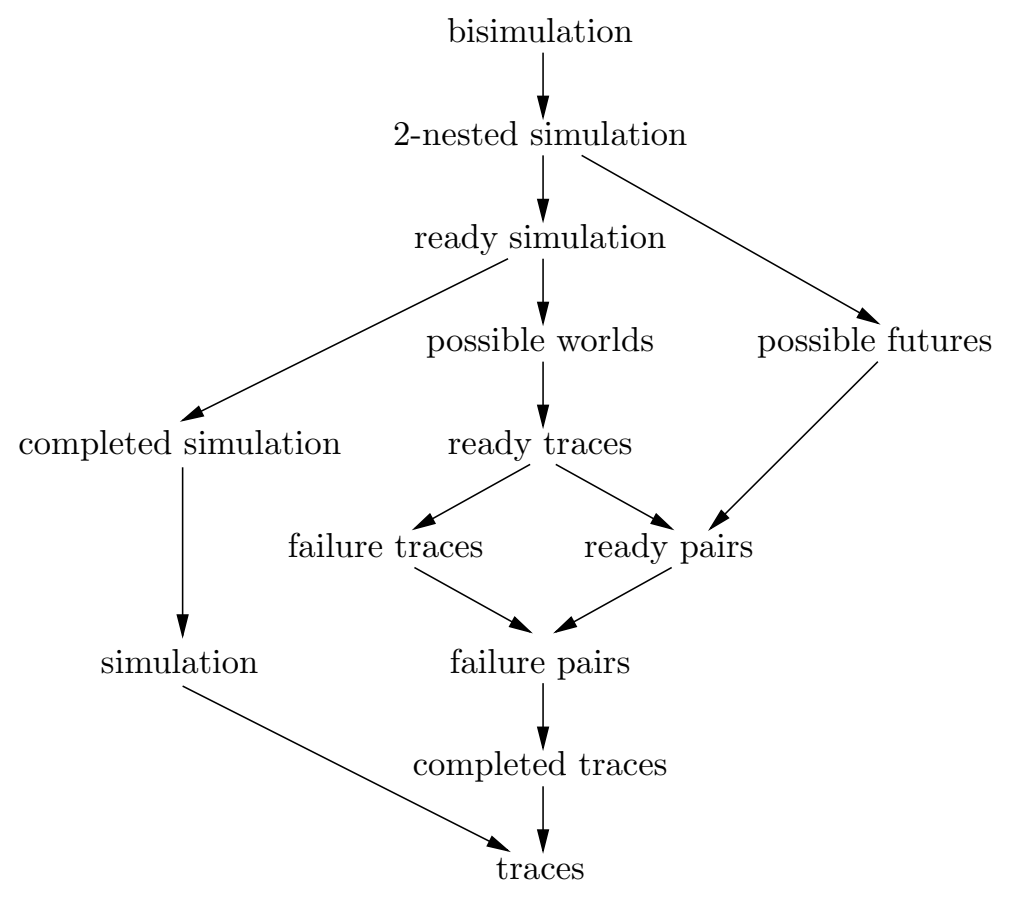

Fig. 1. The linear time - branching time spectrum

perform exactly the same initial actions. Simulation semantics is coarser than completed simulation semantics (meaning that it distinguishes fewer processes), which in turn is coarser than ready simulation semantics. Other semantics in the linear time - branching time spectrum are also based on simulation notions, or on decorated traces. Figure 1 depicts the linear time - branching time spectrum, where a directed edge from one equivalence to another means that the source of the edge is finer than the target.

Van Glabbeek 6] studied the semantics in his spectrum in the setting of the process algebra BCCSP, which contains only basic process algebraic operators from CCS and CSP, but is sufficiently powerful to express all finite synchronization trees. Van Glabbeek gave axiomatizations for the semantics in the spectrum, such that two closed BCCSP terms can be equated by the axioms if and only if they are equivalent.

Having defined a model of an axiomatization for a process algebra in terms of LTSs, it is natural to study the connection between the equations that are valid in the chosen model, and those that are derivable from the axioms using the rules of equational logic. A key question here is whether there is a finite axiomatization that is $\omega$-complete. That is, if all closed instances of an equation can be derived, does this imply that the equation itself can be derived from the axiomatization using the rules of equational logic? (We also refer to an $\omega$-complete axiom system as a basis for the algebra it axiomatizes.) An $\omega$-complete axiomatization of a 
behavioral congruence yields a purely syntactic characterization, independent of LTSs and of the actual details of the definition of the chosen behavioral equivalence, of the semantics of the process algebra. This bridge between syntax and semantics plays an important role in both the practice and the theory of process algebras. From the point of view of practice, these proof systems can be used to perform system verifications in a purely syntactic way using general purpose theorem provers or proof checkers, and form the basis of purpose-built axiomatic verification tools like, e.g., PAM [8].

A notable example of an $\omega$-incomplete axiomatization in the literature is the equational theory of CCS [9]. Therefore laws such as commutativity of parallelism, which are valid in the initial model but which cannot be derived, are often added to the latter equational theory. For such extended equational theories, $\omega$-completeness results were presented in the setting of CCS 10 and $\mathrm{ACP}$ 3.

A number of positive and negative results regarding finite $\omega$-complete axiomatizations for BCCSP occur in the literature. Moller [10] proved that the finite axiomatization for BCCSP modulo bisimulation equivalence is $\omega$-complete. Groote 7. presented a similar result for completed trace equivalence, for trace equivalence (in case of an alphabet with more than one element), and for readiness and failures equivalence (in case of an infinite alphabet). Fokkink and Nain [5] obtained a finite $\omega$-complete axiomatization for BCCSP modulo failures equivalence in case of a finite alphabet, by adding one extra axiom that uses the cardinality of the alphabet. In [4] they proved that in case of a finite alphabet of at least two elements, BCCSP modulo any semantics in between readiness and possible worlds equivalence does not have a finite basis. Blom, Fokkink and Nain 2] proved that in case of an infinite alphabet, BCCSP modulo ready trace equivalence does not have a finite sound and ground-complete axiomatization. Aceto, Fokkink, van Glabbeek and Ingolfsdottir [1] proved a similar negative result for 2-nested simulation and possible futures equivalence, independent of the cardinality of the alphabet 1

In this paper we consider BCCSP modulo completed simulation and ready simulation semantics. We prove that no finite sound and ground-complete axiomatization for BCCSP modulo completed simulation preorder and equivalence is $\omega$-complete. To be more precise, we prove that the infinite family of inequations

$$
a^{n} x \preccurlyeq a^{n} \mathbf{0}+a^{n}(x+y) \quad(n \geq 1)
$$

which are sound modulo completed simulation preorder, cannot be axiomatized in a finite fashion. This result is surprising in the sense that completed simulation is the only semantics in the linear time - branching time spectrum that in case of an infinite alphabet has a finite sound and ground-complete axiomatization for BCCSP, but no finite $\omega$-complete axiomatization.

\footnotetext{
${ }^{1}$ In case of an infinite alphabet, occurrences of action names in axioms should be interpreted as variables, as else most of the axiomatizations mentioned in this paragraph would be infinite.
} 
Next we prove that in case of a finite alphabet $\left\{b_{1}, \ldots, b_{k}\right\}$, no finite sound and ground-complete axiomatization for BCCSP modulo ready simulation preorder and equivalence is $\omega$-complete. To be more precise, we prove that the infinite family of inequations

$$
a^{n} x \preccurlyeq a^{n} \mathbf{0}+a^{n}\left(x+b_{1} \mathbf{0}\right)+\cdots+a^{n}\left(x+b_{k} \mathbf{0}\right) \quad(n \geq 1)
$$

which are sound modulo ready simulation preorder, cannot be axiomatized in a finite fashion.

Finally, we prove, using the technique of inverted substitutions from [7], that in case of an infinite alphabet, the equational theory of BCCSP modulo ready simulation equivalence does have a finite basis.

This paper is set up as follows. Section 2 presents basic definitions regarding simulation semantics, the process algebra BCCSP, and (in)equational logic. Section 3 contains the proofs of the negative results for completed simulation preorder and equivalence. And Section 4 contains the proofs of the negative and positive results for ready simulation preorder and equivalence.

\section{Preliminaries}

Simulation semantics: A labeled transition system contains a set of states, with typical element $s$, and a set of transitions $s \stackrel{a}{\rightarrow} s^{\prime}$, where $a$ ranges over some set $A$ of labels. The set $\mathcal{I}(s)$ consists of those $a \in A$ for which there exists a transition $s \stackrel{a}{\rightarrow} s^{\prime}$.

Definition 1 (Simulation). Assume a labeled transition system.

- A binary relation $R$ on states is a simulation if $s_{0} R s_{1}$ and $s_{0} \stackrel{a}{\rightarrow} s_{0}^{\prime}$ imply $s_{1} \stackrel{a}{\rightarrow} s_{1}^{\prime}$ with $s_{0}^{\prime} R s_{1}^{\prime}$.

- A simulation $R$ is a completed simulation if $s_{0} R s_{1}$ and $\mathcal{I}\left(s_{0}\right)=\emptyset$ imply $\mathcal{I}\left(s_{1}\right)=\emptyset$.

- $A$ simulation $R$ is a ready simulation if $s_{0} R s_{1}$ and $a \notin \mathcal{I}\left(s_{0}\right)$ imply a $\notin$ $\mathcal{I}\left(s_{1}\right)$.

We write $s_{0} \precsim_{\mathrm{CS}} s_{1}$ or $s_{0} \precsim_{R S} s_{1}$ if $s_{0} R s_{1}$ with $R$ a completed or ready simulation, respectively. The kernels of $\precsim_{\mathrm{CS}}$ and $\precsim_{\mathrm{RS}}$ are denoted by $\simeq_{\mathrm{CS}}$ and $\simeq_{\mathrm{RS}}$, respectively.

Syntax of BCCSP: $\operatorname{BCCSP}(A)$ is a basic process algebra for expressing finite process behavior. Its syntax consists of closed (process) terms $p, q$ that are constructed from a constant $\mathbf{0}$, a binary operator ___ called alternative composition, and unary prefix operators $a_{-}$, where $a$ ranges over some nonempty set $A$ of $a c$ tions. Open terms $t, u, v, w$ can moreover contain variables from a countably infinite set $V$ (with typical elements $x, y, z$ ). 
Transition rules: Intuitively, closed $\operatorname{BCCSP}(A)$ terms represent finite process behaviors, where $\mathbf{0}$ does not exhibit any behavior, $p+q$ is the nondeterministic choice between the behaviors of $p$ and $q$, and ap executes action $a$ to transform into $p$. This intuition is captured, in the style of Plotkin, by the transition rules below, which give rise to $A$-labeled transitions between closed terms.

$$
\overline{a x \stackrel{a}{\rightarrow} x} \quad \frac{x \stackrel{a}{\rightarrow} x^{\prime}}{x+y \stackrel{a}{\rightarrow} x^{\prime}} \quad \frac{y \stackrel{a}{\rightarrow} y^{\prime}}{x+y \stackrel{a}{\rightarrow} y^{\prime}}
$$

Completed simulation preorder $\precsim_{\mathrm{CS}}$ and ready simulation preorder $\precsim_{\mathrm{RS}}$ constitute a precongruence for closed $\operatorname{BCCSP}(A)$-terms. That is, $p_{1} \precsim \mathrm{N} q_{1}$ and $p_{2} \precsim_{\mathrm{N}} q_{2}$ implies $a p_{1} \precsim_{\mathrm{N}} a q_{1}$ for $a \in A$ and $p_{1}+p_{2} \precsim_{\mathrm{N}} q_{1}+q_{2}$, where $\mathrm{N}$ ranges over $\{\mathrm{CS}, \mathrm{RS}\}$.

We extend the operational interpretation above to open terms by assuming that variables do not exhibit any behavior. For open terms $t$ and $u$, we define $t \precsim_{\mathrm{N}} u$ (or $t \simeq_{\mathrm{N}} u$ ) if for any closed substitution $\sigma, \sigma(t) \precsim_{\mathrm{N}} \sigma(u)\left(\right.$ or $\sigma(t) \simeq_{\mathrm{N}}$ $\sigma(u)$, respectively).

Equations and inequations: Let axiomatization $E$ be a collection of either inequations $t \preccurlyeq u$ or equations $t \approx u$. We write $E \vdash t \preccurlyeq u$ or $E \vdash t \approx u$ if this (in)equation can be derived from the (in)equations in $E$ using the standard rules of (in)equational logic, where the rule for symmetry can be applied for equational derivations but not for inequational ones. A collection $E$ of (in)equations is sound modulo a preorder $\precsim$ or equivalence $\simeq$ on closed terms if $(E \vdash p \preccurlyeq q) \Rightarrow p \precsim q$ or $(E \vdash p \approx q) \Rightarrow p \simeq q$, respectively, for all closed terms $p$ and $q$. Vice versa, $E$ is ground-complete modulo $\precsim$ or $\simeq$ if $p \precsim q \Rightarrow(E \vdash p \preccurlyeq q)$ or $p \simeq q \Rightarrow(E \vdash p \approx q)$, respectively, for all closed terms $p$ and $q$. Finally, $E$ is $\omega$-complete modulo $\precsim$ or $\simeq$ if $t \precsim u \Rightarrow(E \vdash t \preccurlyeq u)$ or $t \simeq u \Rightarrow(E \vdash t \approx u)$, respectively for all open terms $t$ and $u$.

The core axioms A1-4 [9] for $\operatorname{BCCSP}(A)$ below are $\omega$-complete, and sound modulo bisimulation equivalence, which is the finest semantics in van Glabbeek's linear time - branching time spectrum (see Fig. 1).

$$
\begin{array}{rlrl} 
& \text { A1 } & x+y & \approx y+x \\
& \text { A2 } & (x+y)+z & \approx x+(y+z) \\
& \text { A3 } & x+x & \approx x \\
\text { A4 } & x+\mathbf{0} & \approx x
\end{array}
$$

In the remainder of this paper, process terms are considered modulo A1-2 and A4. A term $x$ or at is a summand of each term $x+u$ or $a t+u$, respectively. We use summation $\sum_{i \in\left\{i_{1}, \ldots, i_{k}\right\}} t_{i}$ (with $k \geq 0$ ) to denote $t_{i_{1}}+\cdots+t_{i_{k}}$, where the empty sum denotes $\mathbf{0}$.

As binding convention, alternative composition and summation bind weaker than prefixing. A (closed) substitution maps variables in $V$ to (closed) terms. For every term $t$ and substitution $\sigma$, the term $\sigma(t)$ is obtained by replacing every occurrence of a variable $x$ in $t$ by $\sigma(x)$. 


\section{Completed Similarity}

In [6], van Glabbeek gave a finite equational axiomatization that is sound and ground-complete for $\operatorname{BCCSP}(A)$ modulo $\simeq_{\mathrm{CS}}$. It consists of axioms A1-4 together with

$$
\mathrm{CS} \quad a(b x+y+z) \approx a(b x+y+z)+a(b x+z)
$$

where $a, b$ range over $A$. Likewise, a finite sound and ground-complete axiomatization for $\operatorname{BCCSP}(A)$ modulo $\precsim_{\mathrm{CS}}$ is obtained by adding $b x+z \precsim_{\mathrm{CS}} b x+y+z$ to A1-4.

In this section we present a proof that the (in)equational theory of $\operatorname{BCCSP}(A)$ modulo completed similarity does not have a finite basis.

\subsection{Completed Simulation Preorder}

We start with proving that the inequational theory of $\operatorname{BCCSP}(A)$ modulo $\precsim$ CS does not have a finite basis. The corner stone for this negative result is the infinite family of inequations

$$
a^{n} x \preccurlyeq a^{n} \mathbf{0}+a^{n}(x+y)
$$

for $n \geq 1$. Here $a^{n} t$ denotes $n$ prefixes of $a: a^{0} t=t$ and $a^{n+1} t=a\left(a^{n} t\right)$. It is not hard to see that these inequations are sound modulo $\precsim$ Cs. The idea is that either $x$ cannot perform any action, in which case $a^{n} x$ is completed simulated by $a^{n} \mathbf{0}$, or $x$ can perform some action, in which case $a^{n} x$ is completed simulated by $a^{n}(x+y)$.

The depth of a term $t$, denoted by depth $(t)$, is the maximal number of transitions in sequence that $t$ can exhibit. It is defined by: $\operatorname{depth}(\mathbf{0})=0, \operatorname{depth}(x)=0$, $\operatorname{depth}(t+u)=\max \{\operatorname{depth}(t), \operatorname{depth}(u)\}$, and $\operatorname{depth}(a t)=\operatorname{depth}(t)+1$.

Proposition 1. Let $E$ be a finite collection of inequations over $\operatorname{BCCSP}(A)$ that is sound modulo $\precsim \mathrm{CS}$. Let $n$ be larger than the depth of any term in $E$. Then from $E$ we cannot derive the inequation

$$
a^{n} x \preccurlyeq a^{n} \mathbf{0}+a^{n}(x+y) .
$$

The main part of this section is devoted to proving Proposition 1, We start with two basic lemmas.

Let $t \stackrel{a_{1} \cdots a_{k}}{\rightarrow} t^{\prime}$ (with $k \geq 0$ ) denote that there is a trace $t=t_{0} \stackrel{a_{1}}{\rightarrow} t_{1} \stackrel{a_{2}}{\rightarrow} \cdots \stackrel{a_{k}}{\rightarrow}$ $t_{k}=t^{\prime}$. If moreover $t^{\prime}=x+t^{\prime \prime}$, then we say that $x$ occurs at depth $k$ in $t$. If $t^{\prime}$ cannot perform any transitions (meaning that each summand of $t^{\prime}$ is a variable or $\mathbf{0})$, then $t \stackrel{a_{1} \cdots a_{k}}{\rightarrow} t^{\prime}$ is called a termination trace of $t$.

Lemma 1. Let $t \precsim \mathrm{CS} u$. If $t_{a_{1} \cdots a_{k}}^{\rightarrow} x+t^{\prime}$, then $u \stackrel{a_{1} \cdots a_{k}}{\rightarrow} x+u^{\prime}$.

Proof. Let $d>\operatorname{depth}(u)$ and $\rho$ a closed substitution such that $\rho(x)=a^{d} \mathbf{0}$ and $\rho(y)=\mathbf{0}$ for any variable $y \neq x$. By assumption, $t \stackrel{a_{1} \cdots a_{k}}{\longrightarrow} x+t^{\prime}$, so $\rho(t) \stackrel{a_{1} \cdots a_{k+d}}{\longrightarrow} \mathbf{0}$ 
(with $a_{k+1} \cdots a_{k+d}=a^{d}$ ). Since $\rho(t) \precsim$ CS $\rho(u)$, it follows that $\rho(u) \stackrel{a_{1} \cdots a_{k+d}}{\rightarrow} v$ with $\mathbf{0} \precsim$ CS $v$, which implies $v \simeq_{\mathrm{CS}} \mathbf{0}$. Since $d>\operatorname{depth}(u)$, clearly $u \stackrel{a_{1} \cdots a_{i}}{\rightarrow} y+u^{\prime}$ where $\rho(y) \stackrel{a_{i+1} \cdots a_{k+d}}{\rightarrow} v$. We have $i \leq \operatorname{depth}(u)<d$, so $\rho(y) \neq \mathbf{0}$, and hence $y=x$ and $i=k$. Concluding, $u \stackrel{a_{1} \cdots a_{k}}{\rightarrow} x+u^{\prime}$.

Lemma 2. If at $\precsim \mathrm{CS} a^{n} \mathbf{0}+a^{n}(x+y)$, then at is completed similar to $a^{n} \mathbf{0}, a^{n} x$, $a^{n} y$ or $a^{n}(x+y)$.

Proof. By assumption, at $\precsim \mathrm{CS} a^{n} \mathbf{0}+a^{n}(x+y)$. Then clearly every termination trace of $t$ has length $n-1$, and executes only $a$ 's. Moreover, by Lemma 1 , $t$ can only contain the variables $x$ and $y$. It follows that for every trace of $t$ such that $t \stackrel{a^{n-1}}{\rightarrow} t^{\prime}, t^{\prime}$ is completed similar to either $\mathbf{0}, x, y$ or $x+y$. Suppose, towards a contradiction, that $t \stackrel{a^{n-1}}{\rightarrow} t_{1}$ and $t \stackrel{a^{n-1}}{\rightarrow} t_{2}$ with $t_{1} \varkappa_{\mathrm{CS}} t_{2}$. In each of the six possible cases (modulo symmetry) we give a closed substitution $\rho$ with $\rho(a t) \not{C C S} \rho\left(a^{n} \mathbf{0}+a^{n}(x+y)\right)$.

- CASES 1,2,3: $t_{1} \simeq_{\mathrm{CS}} \mathbf{0}$ and $t_{2} \simeq_{\mathrm{CS}} x, y$ or $x+y$. Let $\rho(x) \neq_{\mathrm{CS}} \mathbf{0}$ and $\rho(y) \neq_{\mathrm{CS}} \mathbf{0}$. Then $\rho(t) \not{L} \mathrm{CS} a^{n-1} \mathbf{0}$ (because $\rho(t) \stackrel{a^{n-1}}{\rightarrow} \rho\left(t_{2}\right) \neq_{\mathrm{CS}} \mathbf{0}$ ) and $\rho(t) \mathscr{L C S}_{\mathrm{CS}} a^{n-1} \rho(x+y)$ (because $\rho(t) \stackrel{a^{n-1}}{\rightarrow} \rho\left(t_{1}\right) \simeq_{\mathrm{CS}} \mathbf{0}$ and $\left.\rho(x+y) \varkappa_{\mathrm{CS}} \mathbf{0}\right)$.

- CASES 4,5: $t_{1} \simeq_{\mathrm{CS}} x$ and $t_{2} \simeq_{\mathrm{CS}} y$ or $x+y$. Let $\rho(x)=\mathbf{0}$ and $\rho(y) \neq_{\mathrm{CS}}$ 0. Then $\rho(t) \not{L}$ CS $a^{n-1} \mathbf{0}$ (because $\rho(t) \stackrel{a^{n-1}}{\rightarrow} \rho\left(t_{2}\right) \not_{\mathrm{CS}} \mathbf{0}$ ) and $\rho(t) \not{L}_{\mathrm{CS}}$ $a^{n-1} \rho(x+y)$ (because $\rho(t) \stackrel{a^{n-1}}{\rightarrow} \rho\left(t_{1}\right) \simeq_{\mathrm{CS}} \mathbf{0}$ and $\left.\rho(x+y) \neq_{\mathrm{CS}} \mathbf{0}\right)$.

$-\operatorname{CASE} 6: t_{1} \simeq_{\mathrm{CS}} y$ and $t_{2} \simeq_{\mathrm{CS}} x+y$. Let $\rho(x) \neq_{\mathrm{CS}} \mathbf{0}$ and $\rho(y)=\mathbf{0}$. Then $\rho(t) \not{L C S} a^{n-1} \mathbf{0}$ (because $\left.\rho(t) \stackrel{a^{n-1}}{\rightarrow} \rho\left(t_{2}\right) \not_{\mathrm{CS}} \mathbf{0}\right)$ and $\rho(t) \not{L} \mathrm{CS} a^{n-1} \rho(x+y)$ (because $\rho(t) \stackrel{a^{n-1}}{\rightarrow} \rho\left(t_{1}\right) \simeq_{\mathrm{CS}} \mathbf{0}$ and $\left.\rho(x+y) \varkappa_{\mathrm{CS}} \mathbf{0}\right)$.

We conclude that the six cases above all contradict at $\precsim \mathrm{CS} a^{n} \mathbf{0}+a^{n}(x+y)$. Hence it must be the case that for each pair of traces $t \stackrel{a^{n-1}}{\rightarrow} t_{1}$ and $t \stackrel{a^{n-1}}{\rightarrow} t_{2}$, $t_{1} \simeq_{\mathrm{CS}} t_{2}$. Moreover, by Lemma 1 , $t$ does not contain variables at depths smaller than $n-1$. It is not hard to see that this implies the lemma.

The following key lemma paves the way for the proof of Proposition 1 .

Lemma 3. Let $E$ be a finite collection of inequations over $\operatorname{BCCSP}(A)$ that is sound modulo $\precsim_{\mathrm{CS}}$. Let $n$ be greater than the depth of any term in E. Assume that:

$-E \vdash t \preccurlyeq u$;

$-u \precsim \mathrm{CS} a^{n} \mathbf{0}+a^{n}(x+y)$; and

- $t$ has a summand completed similar to $a^{n} x$.

Then $u$ has a summand completed similar to $a^{n} x$. 
Proof. By induction on the depth of the proof of the inequation $t \preccurlyeq u$ from $E$. We proceed by a case analysis on the last rule used in the proof of $t \preccurlyeq u$ from $E$.

- CASE 1: $E \vdash t \preccurlyeq u$ because $\sigma(v)=t$ and $\sigma(w)=u$ for some $v \preccurlyeq w \in E$ and substitution $\sigma$.

Since $t=\sigma(v)$ has a summand completed similar to $a^{n} x$, we can distinguish two cases.

- CASE 1.1: $v$ has as summand some variable $z$ where $\sigma(z)$ has a summand completed similar to $a^{n} x$.

Since $v$ has $z$ as summand, and soundness of $E$ yields $v \precsim$ Cs $w$, by Lemma 1, $w$ also has $z$ as summand. Then clearly $u=\sigma(w)$ has a summand completed similar to $a^{n} x$.

- CASE 1.2: $v$ has a summand $a v^{\prime}$ where $\sigma\left(a v^{\prime}\right) \simeq_{\mathrm{CS}} a^{n} x$.

Since $n$ is larger than the depth of $v, \operatorname{depth}\left(a v^{\prime}\right)<n$. So, since $\sigma\left(a v^{\prime}\right) \simeq_{\mathrm{CS}}$ $a^{n} x, a v^{\prime} \stackrel{a^{k}}{\rightarrow} z+v^{\prime \prime}$ where $1 \leq k<n$ and $\sigma(z) \simeq_{\mathrm{CS}} a^{n-k} x$. Since $v \precsim \mathrm{CS} w$, by Lemma 1, $w$ has a summand $a w^{\prime}$ such that $w^{\prime} \stackrel{a^{k-1}}{\rightarrow} z+w^{\prime \prime}$, and consequently $\sigma\left(w^{\prime}\right) \stackrel{a^{n-1}}{\rightarrow} w^{\prime \prime \prime}$ with $w^{\prime \prime \prime} \simeq_{\mathrm{CS}} x$. Furthermore, $a \sigma\left(w^{\prime}\right) \precsim \mathrm{CS}$ $\sigma(w) \precsim \mathrm{CS} a^{n} \mathbf{0}+a^{n}(x+y)$. Then Lemma 2 yields $\sigma\left(w^{\prime}\right) \simeq_{\mathrm{CS}} a^{n-1} x$. Hence $\sigma\left(a w^{\prime}\right) \simeq{ }_{\mathrm{CS}} a^{n} x$. So $u=\sigma(w)$ has a summand completed similar to $a^{n} x$.

- CASE 2: $E \vdash t \preccurlyeq u$ by reflexivity. Then $t=u$, so $u$ trivially has a summand completed similar to $a^{n} x$.

- CASE 3: $E \vdash t \preccurlyeq u$ by transitivity.

Then $E \vdash t \preccurlyeq v$ and $E \vdash v \preccurlyeq u$ for some term $v$. By the soundness of $E$, $v \precsim \mathrm{CS} u \precsim \mathrm{CS} a^{n} \mathbf{0}+a^{n}(x+y)$. So by induction, $v$ has a summand completed similar to $a^{n} x$. Hence, again by induction, $u$ has a summand completed similar to $a^{n} x$.

- CASE 4: $E \vdash t \preccurlyeq u$ because $t=t^{\prime}+t^{\prime \prime}$ and $u=u^{\prime}+u^{\prime \prime}$ for some $t^{\prime}, u^{\prime}, t^{\prime \prime}, u^{\prime \prime}$ such that $E \vdash t^{\prime} \preccurlyeq u^{\prime}$ and $E \vdash t^{\prime \prime} \preccurlyeq u^{\prime \prime}$.

Since $t$ has a summand completed similar to $a^{n} x$, so does either $t^{\prime}$ or $t^{\prime \prime}$. Assume, without loss of generality, that $t^{\prime}$ has a summand completed similar to $a^{n} x$. Then clearly $u^{\prime} \neq_{\mathrm{CS}} \mathbf{0}$. So, since $u \precsim_{\mathrm{CS}} a^{n} \mathbf{0}+a^{n}(x+y)$, it follows that $u^{\prime} \precsim \mathrm{CS} a^{n} \mathbf{0}+a^{n}(x+y)$. By induction, $u^{\prime}$ (and thus $u$ ) has a summand completed similar to $a^{n} x$.

- CASE 5: $E \vdash t \preccurlyeq u$ because $t=a t^{\prime}$ and $u=a u^{\prime}$ for some $t^{\prime}, u^{\prime}$ such that $E \vdash t^{\prime} \preccurlyeq u^{\prime}$.

Since $t=a t^{\prime}$ consists of a single summand, $a t^{\prime} \simeq_{\mathrm{CS}} a^{n} x$. By the soundness of $E, a^{n} x \precsim \mathrm{CS} a u^{\prime}$. Since moreover $a u^{\prime} \precsim \mathrm{CS} a^{n} \mathbf{0}+a^{n}(x+y)$, Lemma 2 yields $u=a u^{\prime} \simeq_{\mathrm{CS}} a^{n} x$.

Now we are in a position to prove Proposition [1]

Proof. Let $E$ be a finite collection of inequations over $\operatorname{BCCSP}(A)$ that is sound modulo $\precsim$ CS . Let $n$ be larger than the depth of any term in $E$. 
$a^{n} \mathbf{0}+a^{n}(x+y)$ does not contain a summand completed similar to $a^{n} x$. So according to Lemma 3, the inequation $a^{n} x \preccurlyeq a^{n} \mathbf{0}+a^{n}(x+y)$, which is sound modulo $\precsim$ CS, cannot be derived from $E$.

Theorem 1. $\mathrm{CS}$ is not finitely based over $\operatorname{BCCSP}(A)$.

Proof. By Proposition 1, no finite collection of inequations over $\operatorname{BCCSP}(A)$ that is sound modulo $\precsim \mathrm{CS}$ proves all inequations that are sound modulo $\precsim \mathrm{CS}$.

\subsection{Completed Simulation Equivalence}

Following the same line as in Section 3.1, we can prove that the equational theory of $\operatorname{BCCSP}(A)$ modulo $\simeq_{\mathrm{CS}}$ does not have a finite basis. The proofs are similar to the proofs of the corresponding results in the previous section.

Lemma 4. Let $E$ be a finite collection of equations over $\operatorname{BCCSP}(A)$ that is sound modulo $\simeq_{\mathrm{CS}}$. Let $n$ be greater than the depth of any term in $E$. Assume that:

$-E \vdash t \approx u$

$-u \simeq_{\mathrm{CS}} a^{n} \mathbf{0}+a^{n}(x+y) ;$ and

- $t$ has a summand completed similar to $a^{n} x$.

Then $u$ has a summand completed similar to $a^{n} x$.

Proof. By induction on the depth of the proof of the equation $t \approx u$ from $E$. First note that by postulating that for each axiom in $E$ also its symmetric counterpart is present in $E$, one may assume that, without loss of generality, applications of symmetry happen first in equational proof. Thus in the proof, we can tacitly assume that equational axiomatization $E$ is closed with respect to symmetry.

Now the proof proceeds by a case analysis on the last rule used in the proof of $t \approx u$ from $E$, similar to the proof of Lemma 3. This case analysis is omitted here.

Proposition 2. Let $E$ be a finite collection of equations over $\operatorname{BCCSP}(A)$ that is sound modulo $\simeq_{\mathrm{CS}}$. Let $n$ be larger than the depth of any term in $E$. Then from $E$ we cannot derive the equation

$$
a^{n} x+a^{n} \mathbf{0}+a^{n}(x+y) \approx a^{n} \mathbf{0}+a^{n}(x+y)
$$

Proof. $a^{n} \mathbf{0}+a^{n}(x+y)$ does not contain a summand completed similar to $a^{n} x$. So according to Lemma 4, the equation $a^{n} x+a^{n} \mathbf{0}+a^{n}(x+y) \approx a^{n} \mathbf{0}+a^{n}(x+y)$, which is sound modulo $\simeq_{\mathrm{CS}}$, cannot be derived from $E$.

Theorem 2. $\simeq_{\mathrm{CS}}$ is not finitely based over $\operatorname{BCCSP}(A)$.

Proof. By Proposition 2, no finite collection of equations over $\operatorname{BCCSP}(A)$ that is sound modulo $\simeq_{\mathrm{CS}}$ proves all equations that are sound modulo $\simeq_{\mathrm{CS}}$. 


\section{Ready Similarity}

Blom, Fokkink and Nain 2] gave a finite equational axiomatization that is sound and ground-complete for $\operatorname{BCCSP}(A)$ modulo $\simeq_{\mathrm{RS}}$. It consists of axioms $\mathrm{A} 1-4$ together with

$$
\mathrm{RS} \quad a(b x+b y+z) \approx a(b x+b y+z)+a(b x+z)
$$

where $a, b$ range over $A$. If $A$ is infinite, then Groote's technique of inverted substitutions from [7] can be applied in a straightforward fashion to prove that this axiomatization is $\omega$-complete. So in this case, ready simulation equivalence is finitely based over $\operatorname{BCCSP}(A)$. This finite basis can be adapted in a straightforward fashion to a finite basis for $\operatorname{BCCSP}(A)$ modulo ready simulation preorder (simply add $b x+z \precsim \mathrm{RS} b x+b y+z$ to A1-4).

In this section we prove that if $A$ is finite, then ready simulation preorder and equivalence are not finitely based over $\operatorname{BCCSP}(A)$. The infinite family of equations, and the structure of the proof, are very similar to the case of completed similarity in the previous section (where we obtained a negative result for arbitrary alphabets).

\subsection{Ready Simulation Preorder with $|A|<\infty$}

First we present a proof that if $A$ is finite, then the inequational theory of $\operatorname{BCCSP}(A)$ modulo $\precsim_{\mathrm{RS}}$ does not have a finite basis. The corner stone for this negative result is the infinite family of inequations

$$
a^{n} x \preccurlyeq a^{n} \mathbf{0}+\sum_{b \in A} a^{n}(x+b \mathbf{0})
$$

for $n \geq 1$. It is not hard to see that these inequations are sound modulo $\precsim$ Rs. The idea is that either $x$ cannot perform any action, in which case $a^{n} x$ is ready simulated by $a^{n} \mathbf{0}$, or $x$ can perform some action $b$, in which case $a^{n} x$ is ready simulated by $a^{n}(x+b \mathbf{0})$.

Proposition 3. Let $|A|<\infty$. Let $E$ be a finite collection of inequations over $\operatorname{BCCSP}(A)$ that is sound modulo $\precsim \mathrm{RS}$. Let $n$ be larger than the depth of any term in $E$. Then from $E$ we cannot derive the inequation

$$
a^{n} x \preccurlyeq a^{n} \mathbf{0}+\sum_{b \in A} a^{n}(x+b \mathbf{0}) .
$$

The main part of this section is devoted to proving Proposition 3 . Note that Lemma 1 also applies to ready simulation preorder, as it is finer than completed simulation preorder.

Lemma 5. Let $|A|<\infty$. If at $\precsim \mathrm{RS} a^{n} \mathbf{0}+\sum_{b \in A} a^{n}(x+b \mathbf{0})$, then at is ready similar to $a^{n} \mathbf{0}, a^{n} x$ or $a^{n}(x+b \mathbf{0})$ for some $b \in A$. 
Proof. By assumption, at $\precsim \mathrm{RS} a^{n} \mathbf{0}+\sum_{b \in A} a^{n}(x+b \mathbf{0})$. Then clearly every termination trace of $t$ is of the form $t \stackrel{a^{n-1}}{\rightarrow} t^{\prime}$ or $t \stackrel{a^{n-1} b}{\rightarrow} t^{\prime}$. Moreover, by Lemma 1 , $t$ can only contain the variable $x$, and $x$ cannot occur at depth $n$ in $t$. It follows that for every trace of $t$ such that $t \stackrel{a^{n-1}}{\rightarrow} t^{\prime}, t^{\prime}$ is ready similar to either $\mathbf{0}, x$ or $x+b_{0} \mathbf{0}$ for some $b_{0} \in A$. Suppose, towards a contradiction, that $t \stackrel{a^{n-1}}{\rightarrow} t_{1}$ and $t \stackrel{a^{n-1}}{\rightarrow} t_{2}$ with $t_{1} \neq_{\mathrm{RS}} t_{2}$. In each of the four possible cases (modulo symmetry) we give a closed substitution $\rho$ with $\rho(a t) \mathscr{L}_{\mathrm{RS}} \rho\left(a^{n} \mathbf{0}+\sum_{b \in A} a^{n}(x+b \mathbf{0})\right)$.

- CASES $1,2: t_{1} \simeq_{\mathrm{RS}} \mathbf{0}$ and $t_{2} \simeq_{\mathrm{RS}} x$ or $x+b_{0} \mathbf{0}$ for some $b_{0} \in A$. Let $\rho(x) \neq_{\mathrm{RS}}$

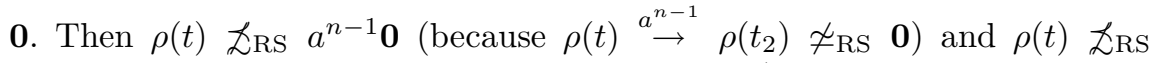
$a^{n-1} \rho(x+b \mathbf{0})$ for each $b \in A$ (because $\rho(t) \stackrel{a^{n-1}}{\rightarrow} \rho\left(t_{1}\right) \simeq_{\mathrm{RS}} \mathbf{0}$ and $\rho(x+$ b0) $\left.\neq_{\mathrm{RS}} \mathbf{0}\right)$.

- CASE 3: $t_{1} \simeq_{\mathrm{RS}} x$ and $t_{2} \simeq_{\mathrm{RS}} x+b_{0} \mathbf{0}$ for some $b_{0} \in A$. Let $\rho(x)=\mathbf{0}$. Then

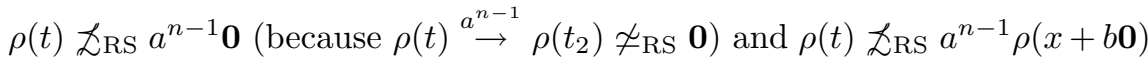
for each $b \in A$ (because $\rho(t) \stackrel{a^{n-1}}{\rightarrow} \rho\left(t_{1}\right) \simeq_{\mathrm{RS}} \mathbf{0}$ and $\left.\rho(x+b \mathbf{0}) \neq_{\mathrm{RS}} \mathbf{0}\right)$.

- CASE 4: $t_{1} \simeq_{\mathrm{RS}} x+b_{0} \mathbf{0}$ and $t_{2} \simeq_{\mathrm{RS}} x+b_{1} \mathbf{0}$ for some $b_{0}, b_{1} \in A$ with $b_{0} \neq b_{1}$. Let $\rho(x)=\mathbf{0}$. Then $\rho(t) \mathscr{L}_{\mathrm{RS}} a^{n-1} \mathbf{0}$ (because $\rho(t) \stackrel{a^{n-1}}{\rightarrow} \rho\left(t_{1}\right) \neq_{\mathrm{RS}} \mathbf{0}$ ) and $\rho(t) \swarrow_{\mathrm{RS}} a^{n-1} \rho(x+b \mathbf{0})$ for each $b \in A$ (because $b \neq b_{i}$ for $i=0$ or $i=1$, so that $\rho(t) \stackrel{a^{n-1}}{\rightarrow} \rho\left(t_{i}\right) \simeq_{\mathrm{RS}} b_{i} \mathbf{0}$ and $\left.\rho(x+b \mathbf{0}) \neq_{\mathrm{RS}} b_{i} \mathbf{0}\right)$.

We conclude that the four cases above all contradict $a t \precsim_{\mathrm{RS}} a^{n} \mathbf{0}+\sum_{b \in A} a^{n}(x+$ b0). Hence it must be the case that for each pair of traces $t \stackrel{a^{n-1}}{\rightarrow} t_{1}$ and $t \stackrel{a^{n-1}}{\rightarrow} t_{2}$, $t_{1} \simeq_{\mathrm{RS}} t_{2}$. Moreover, by Lemma 1, $t$ does not contain variables at depths smaller than $n-1$. It is not hard to see that this implies the lemma.

The following key lemma paves the way for the proof of Proposition 3 ,

Lemma 6. Let $|A|<\infty$. Let $E$ be a finite collection of inequations over BCCSP (A) that is sound modulo $\precsim \mathrm{RS}$. Let $n$ be greater than the depth of any term in E. Assume that:

$-E \vdash t \preccurlyeq u$;

$-u \precsim_{\mathrm{RS}} a^{n} \mathbf{0}+\sum_{b \in A} a^{n}(x+b \mathbf{0}) ;$ and

- $t$ has a summand ready similar to $a^{n} x$.

Then $u$ has a summand ready similar to $a^{n} x$.

Proof. By induction on the depth of the proof of the inequation $t \preccurlyeq u$ from $E$. We proceed by a case analysis on the last rule used in the proof of $t \preccurlyeq u$ from $E$.

- CASE 1: $E \vdash t \preccurlyeq u$ because $\sigma(v)=t$ and $\sigma(w)=u$ for some $v \preccurlyeq w \in E$ and substitution $\sigma$.

Since $t=\sigma(v)$ has a summand ready similar to $a^{n} x$, we can distinguish two cases. 
- CASE 1.1: $v$ has as summand some variable $z$ where $\sigma(z)$ has a summand ready similar to $a^{n} x$.

Since $v$ has $z$ as summand, and soundness of $E$ yields $v \precsim$ Rs $w$, by Lemma 1, $w$ also has $z$ as summand. Then clearly $u=\sigma(w)$ has a summand ready similar to $a^{n} x$.

- CASE 1.2: $v$ has a summand $a v^{\prime}$ where $\sigma\left(a v^{\prime}\right) \simeq_{\mathrm{RS}} a^{n} x$.

Since $n$ is larger than the depth of $v, \operatorname{depth}\left(a v^{\prime}\right)<n$. So, since $\sigma\left(a v^{\prime}\right) \simeq_{\mathrm{RS}}$ $a^{n} x, a v^{\prime} \stackrel{a^{k}}{\rightarrow} z+v^{\prime \prime}$ where $1 \leq k<n$ and $\sigma(z) \simeq_{\mathrm{RS}} a^{n-k} x$. Since $v \precsim$ RS $w$, by Lemma 1, $w$ has a summand $a w^{\prime}$ such that $w^{\prime} \stackrel{a^{k-1}}{\rightarrow}$ $z+w^{\prime \prime}$, and consequently $\sigma\left(w^{\prime}\right) \stackrel{a^{n-1}}{\rightarrow} w^{\prime \prime \prime}$ with $w^{\prime \prime \prime} \simeq_{\mathrm{RS}} x$. Furthermore, $a \sigma\left(w^{\prime}\right) \precsim_{\mathrm{RS}} \sigma(w) \precsim_{\mathrm{RS}} a^{n} \mathbf{0}+\sum_{b \in A} a^{n}(x+b \mathbf{0})$. Then Lemma 5 yields $\sigma\left(w^{\prime}\right) \simeq_{\mathrm{RS}} a^{n-1} x$. Hence $\sigma\left(a w^{\prime}\right) \simeq_{\mathrm{RS}} a^{n} x$. So $u=\sigma(w)$ has a summand ready similar to $a^{n} x$.

- CASE 2: $E \vdash t \preccurlyeq u$ by reflexivity. Then $t=u$, so $u$ trivially has a summand ready similar to $a^{n} x$.

- CASE 3: $E \vdash t \preccurlyeq u$ by transitivity.

Then $E \vdash t \preccurlyeq v$ and $E \vdash v \preccurlyeq u$ for some term $v$. By the soundness of $E$, $v \precsim \mathrm{RS} u \precsim_{\mathrm{RS}} a^{n} \mathbf{0}+\sum_{b \in A} a^{n}(x+b \mathbf{0})$. So by induction, $v$ has a summand ready similar to $a^{n} x$. Hence, again by induction, $u$ has a summand ready similar to $a^{n} x$.

- CASE 4: $E \vdash t \preccurlyeq u$ because $t=t^{\prime}+t^{\prime \prime}$ and $u=u^{\prime}+u^{\prime \prime}$ for some $t^{\prime}, u^{\prime}, t^{\prime \prime}, u^{\prime \prime}$ such that $E \vdash t^{\prime} \preccurlyeq u^{\prime}$ and $E \vdash t^{\prime \prime} \preccurlyeq u^{\prime \prime}$.

Since $t$ has a summand ready similar to $a^{n} x$, so does either $t^{\prime}$ or $t^{\prime \prime}$. Assume, without loss of generality, that $t^{\prime}$ has a summand ready similar to $a^{n} x$. Then clearly $u^{\prime} \neq_{\mathrm{RS}} \mathbf{0}$. So, since $u \precsim_{\mathrm{RS}} a^{n} \mathbf{0}+\sum_{b \in A} a^{n}(x+b \mathbf{0})$, it follows that $u^{\prime} \precsim_{\mathrm{RS}} a^{n} \mathbf{0}+\sum_{b \in A} a^{n}(x+b \mathbf{0})$. By induction, $u^{\prime}$ (and thus $u$ ) has a summand ready similar to $a^{n} x$.

- CASE 5: $E \vdash t \preccurlyeq u$ because $t=a t^{\prime}$ and $u=a u^{\prime}$ for some $t^{\prime}, u^{\prime}$ such that $E \vdash t^{\prime} \preccurlyeq u^{\prime}$.

Since $t=a t^{\prime}$ consists of a single summand, $a t^{\prime} \simeq_{\mathrm{RS}} a^{n} x$. By the soundness of $E, a^{n} x \precsim_{\mathrm{RS}} a u^{\prime}$. Since moreover $a u^{\prime} \precsim_{\mathrm{RS}} a^{n} \mathbf{0}+\sum_{b \in A} a^{n}(x+b \mathbf{0})$, Lemma 5 yields $u=a u^{\prime} \simeq_{\mathrm{RS}} a^{n} x$.

Now we are in a position to prove Proposition 3

Proof. Let $E$ be a finite collection of inequations over $\operatorname{BCCSP}(A)$ that is sound modulo $\precsim$ Rs. Let $n$ be larger than the depth of any term in $E$.

$a^{n} \mathbf{0}+\sum_{b \in A} a^{n}(x+b \mathbf{0})$ does not contain a summand ready similar to $a^{n} x$. So according to Lemma 6, the inequation $a^{n} x \preccurlyeq a^{n} \mathbf{0}+\sum_{b \in A} a^{n}(x+b \mathbf{0})$, which is sound modulo $\precsim$ RS, cannot be derived from $E$.

Theorem 3. Let $|A|<\infty$. Then $\precsim \mathrm{RS}$ is not finitely based over $\operatorname{BCCSP}(A)$.

Proof. By Proposition 3, no finite collection of inequations over $\operatorname{BCCSP}(A)$ that is sound modulo $\precsim$ Rs proves all inequations that are sound modulo $\precsim$ Rs . 


\subsection{Ready Simulation Equivalence with $|A|<\infty$}

Following the same line as in Section 4.1, we can prove that if $A$ is finite, then the equational theory of $\operatorname{BCCSP}(A)$ modulo $\simeq_{\mathrm{RS}}$ does not have a finite basis. The proofs are similar to the proofs of the corresponding results in the previous section.

Lemma 7. Let $|A|<\infty$. Let $E$ be a finite collection of equations over BCCSP (A) that is sound modulo $\simeq_{\mathrm{RS}}$. Let $n$ be greater than the depth of any term in E. Assume that:

$-E \vdash t \approx u$

$-u \simeq_{\mathrm{RS}} a^{n} \mathbf{0}+\sum_{b \in A} a^{n}(x+b \mathbf{0}) ;$ and

- $t$ has a summand ready similar to $a^{n} x$.

Then $u$ has a summand ready similar to $a^{n} x$.

Proof. By induction on the depth of the proof of the equation $t \approx u$ from $E$. Recall that as in the proof of Lemma 4 without loss of generality, we may assume that applications of symmetry happen first in equational proof, i.e. $E$ is closed with respect to symmetry.

Now the proof proceeds by a case analysis on the last rule used in the proof of $t \approx u$ from $E$, similar to the proof of Lemma 6 . This case analysis is omitted here.

Proposition 4. Let $|A|<\infty$. Let $E$ be a finite collection of equations over $\operatorname{BCCSP}(A)$ that is sound modulo $\simeq_{\mathrm{RS}}$. Let $n$ be larger than the depth of any term in $E$. Then from $E$ we cannot derive the equation

$$
a^{n} x+a^{n} \mathbf{0}+\sum_{b \in A} a^{n}(x+b \mathbf{0}) \approx a^{n} \mathbf{0}+\sum_{b \in A} a^{n}(x+b \mathbf{0})
$$

Proof. Let $E$ be a finite collection of equations over $\operatorname{BCCSP}(A)$ that is sound modulo $\simeq_{\mathrm{RS}}$. Let $n$ be larger than the depth of any term in $E$.

$a^{n} \mathbf{0}+\sum_{b \in A} a^{n}(x+b \mathbf{0})$ does not contain a summand ready similar to $a^{n} x$. So according to Lemma 7 the equation $a^{n} x+a^{n} \mathbf{0}+\sum_{b \in A} a^{n}(x+b \mathbf{0}) \approx a^{n} \mathbf{0}+$ $\sum_{b \in A} a^{n}(x+b \mathbf{0})$, which is sound modulo $\simeq_{\mathrm{RS}}$, cannot be derived from $E$.

Theorem 4. Let $|A|<\infty$. Then $\simeq_{\mathrm{RS}}$ is not finitely based over $\operatorname{BCCSP}(A)$.

Proof. By Proposition 4, no finite collection of equations over $\operatorname{BCCSP}(A)$ that is sound modulo $\simeq_{\mathrm{RS}}$ proves all equations that are sound modulo $\simeq_{\mathrm{RS}}$.

\subsection{Ready Simulation Equivalence with $|A|=\infty$}

In this section we prove that if $A$ is infinite, then the axiomatization A1-4 together with

$$
\mathrm{RS} \quad a(b x+b y+z) \approx a(b x+b y+z)+a(b x+z)
$$


from [2], which is ground-complete for $\operatorname{BCCSP}(A)$ modulo $\simeq_{\mathrm{RS}}$, is $\omega$-complete. The proof is based on inverted substitutions; this technique, which is due to Groote [7], works as follows. Consider an axiomatization $E$. For each equation $t \approx u$ of which all closed instances can be derived from $E$, one must define a closed substitution $\rho$ and a mapping $R: \mathrm{T}(\mathrm{BCCSP}) \rightarrow \mathbb{T}(\mathrm{BCCSP})$ such that:

(1) $E \vdash R(\rho(t)) \approx t$ and $E \vdash R(\rho(u)) \approx u$;

(2) for each function symbol $f$ (with arity $n$ ), $E \cup\left\{p_{i} \approx q_{i}, R\left(p_{i}\right) \approx R\left(q_{i}\right)\right.$ $i=1, \ldots, n\} \vdash R\left(f\left(p_{1}, \ldots, p_{n}\right)\right) \approx R\left(f\left(q_{1}, \ldots, q_{n}\right)\right)$ for all closed terms $p_{1}, \ldots, p_{n}, q_{1}, \ldots, q_{n} ;$ and

(3) $E \vdash R(\sigma(v)) \approx R(\sigma(w))$ for each $v \approx w \in E$ and closed substitution $\sigma$.

Then, as proved in [7], $E$ is $\omega$-complete.

Theorem 5. If $|A|=\infty$, then $A 1-4+R S$ is $\omega$-complete.

Proof. Consider two terms $t, u \in \mathbb{T}$ (BCCSP). Define $\rho: V \rightarrow \mathrm{T}$ (BCCSP) by $\rho(x)=a_{x} \mathbf{0}$, where $a_{x}$ is a unique action for $x \in V$ that occurs in neither $t$ nor $u$. Such actions exist because $A$ is infinite. We define $R: \mathrm{T}(\mathrm{BCCSP}) \rightarrow \mathbb{T}$ (BCCSP) as follows:

$$
\left\{\begin{array}{l}
R(\mathbf{0})=\mathbf{0} \\
R(a t)=a R(t) \text { if } a \neq a_{x} \text { for all } x \in V \\
R\left(a_{x} t\right)=x \\
R(t+u)=R(t)+R(u)
\end{array}\right.
$$

We now check the three properties from [7]:

(1) Since $t$ and $u$ do not contain actions of the form $a_{x}$, clearly $R(\rho(t))=t$.

(2) Consider the operator _ $+\ldots$. From $R\left(p_{1}\right) \approx R\left(q_{1}\right)$ and $R\left(p_{2}\right) \approx R\left(q_{2}\right)$ we derive $R\left(p_{1}+p_{2}\right)=R\left(p_{1}\right)+R\left(p_{2}\right) \approx R\left(q_{1}\right)+R\left(q_{2}\right)=R\left(q_{1}+q_{2}\right)$.

Consider the prefix operator $a_{-}$. We distinguish two cases.

- $a \neq a_{y}$ for all $y \in V$. Then from $R\left(p_{1}\right) \approx R\left(q_{1}\right)$ we derive $R\left(a p_{1}\right)=$ $a R\left(p_{1}\right) \approx a R\left(q_{1}\right)=R\left(a q_{1}\right)$.

- $a=a_{y}$ for some $y \in V$. Then $R\left(a_{y} p_{1}\right)=y=R\left(a_{y} q_{1}\right)$.

(3) For A1-4, the proof is trivial. We check the remaining case RS. Let $\sigma$ be a closed substitution. We consider three cases.

- $a=a_{y}$ for some $y \in V$.

Then $R\left(a_{y}\left(b \sigma\left(x_{1}\right)+b \sigma\left(x_{2}\right)+\sigma\left(x_{3}\right)\right)\right)=y \approx y+y=R\left(a_{y}\left(b \sigma\left(x_{1}\right)+\right.\right.$ $\left.\left.b \sigma\left(x_{2}\right)+\sigma\left(x_{3}\right)\right)+a_{y}\left(b \sigma\left(x_{1}\right)+\sigma\left(x_{3}\right)\right)\right)$.

- $a \neq a_{y}$ for all $y \in V$ and $b=b_{z}$ for some $z \in V$.

Then $R\left(a\left(b_{z} \sigma\left(x_{1}\right)+b_{z} \sigma\left(x_{2}\right)+\sigma\left(x_{3}\right)\right)\right)=a\left(z+z+R\left(\sigma\left(x_{3}\right)\right)\right) \approx a(z+$ $\left.z+R\left(\sigma\left(x_{3}\right)\right)\right)+a\left(z+R\left(\sigma\left(x_{3}\right)\right)\right)=R\left(a\left(b_{z} \sigma\left(x_{1}\right)+b_{z} \sigma\left(x_{2}\right)+\sigma\left(x_{3}\right)\right)+\right.$ $\left.a\left(b_{z} \sigma\left(x_{1}\right)+\sigma\left(x_{3}\right)\right)\right)$.

- $a \neq a_{y}$ for all $y \in V$ and $b \neq b_{z}$ for all $z \in V$.

Then $R\left(a\left(b \sigma\left(x_{1}\right)+b \sigma\left(x_{2}\right)+\sigma\left(x_{3}\right)\right)\right)=a\left(b R\left(\sigma\left(x_{1}\right)\right)+b R\left(\sigma\left(x_{2}\right)\right)+R\left(\sigma\left(x_{3}\right)\right)\right)$ $\approx a\left(b R\left(\sigma\left(x_{1}\right)\right)+b R\left(\sigma\left(x_{2}\right)\right)+R\left(\sigma\left(x_{3}\right)\right)\right)+a\left(b R\left(\sigma\left(x_{1}\right)\right)+R\left(\sigma\left(x_{3}\right)\right)\right)=$ $R\left(a\left(b \sigma\left(x_{1}\right)+b \sigma\left(x_{2}\right)+\sigma\left(x_{3}\right)\right)+a\left(b \sigma\left(x_{1}\right)+\sigma\left(x_{3}\right)\right)\right)$. 


\section{References}

1. L. Aceto, W.J. Fokkink, R.J. van Glabbeek, and A. Ingolfsdottir. Nested semantics over finite trees are equationally hard. Information and Computation, 191(2): 203-232, 2004.

2. S.C.C. Blom, W.J. Fokkink, and S. Nain. On the axiomatizability of ready traces, ready simulation and failure traces. In Proceedings 30th Colloquium on Automata, Languages and Programming (ICALP'03), Eindhoven, LNCS 2719, pp. 109-118. Springer, 2003.

3. W.J. Fokkink and S.P. Luttik. An $\omega$-complete equational specification of interleaving. In Proceedings 27th Colloquium on Automata, Languages and Programming (ICALP'00), Geneva, LNCS 1853, pp. 729-743. Springer, 2000.

4. W.J. Fokkink and S. Nain. On finite alphabets and infinite bases: From ready pairs to possible worlds. In Proceedings 7th Conference on Foundations of Software Science and Computation Structures (FOSSACS'04), Barcelona, LNCS 2987, pp. 182-194. Springer, 2004.

5. W.J. Fokkink and S. Nain. A finite basis for failure semantics. In Proceedings 32nd Colloquium on Automata, Languages and Programming (ICALP'05), Lisbon, LNCS 3580, pp. 755-765. Springer, 2005.

6. R.J. van Glabbeek. The linear time - branching time spectrum I. The semantics of concrete, sequential processes. In J.A. Bergstra, A. Ponse, and S.A. Smolka, eds, Handbook of Process Algebra, pp. 3-99. Elsevier, 2001.

7. J.F. Groote. A new strategy for proving $\omega$-completeness with applications in process algebra. In Proceedings 1st Conference on Concurrency Theory (CONCUR'90), Amsterdam, LNCS 458, pp. 314-331. Springer, 1990.

8. H. Lin. PAM: A process algebra manipulator. Formal Methods in System Design, 7(3):243-259, 1995.

9. R. Milner. Communication and Concurrency. Prentice Hall, 1989.

10. F. Moller. Axioms for Concurrency. PhD thesis, University of Edinburgh, 1989. 